\title{
News Application Adaptation based on User Sensory Profile
}

\author{
Roxana Agrigoroaie, Stefan Dan Ciocirlan, and Adriana Tapus \\ Autonomous Systems and Robotics Laboratory, U2IS \\ ENSTA-ParisTech, Université Paris-Saclay, Palaiseau, France \\ firstname.lastname@ensta-paristech.fr
}

\begin{abstract}
The EU H2020 ENRICHME project is aimed at developing a socially assistive robot that can adapt its behavior based on the profile of the individual it interacts with. In this paper, we investigate how the ENRICHME project web-based News Application can be adapted based on the sensory profile and personality of the individual. The interaction between the robot and the individual takes place at three different distances $(70 \mathrm{~cm}, 1.2 \mathrm{~m}$, and $2 \mathrm{~m})$. The robot can use visual (showing or not showing the news on its touchscreen) and auditory (reading the news out loud) stimuli during the interaction. We looked at different physiological parameters (blinking, heart rate, respiration rate, and GSR) and we can report that we found statistical results that show that the physiological parameters vary based on interaction distance, condition, sensory profile, and personality.
\end{abstract}

\section{Introduction}

The main purpose of the EU H2020 ENRICHME ${ }^{1}$ project is to develop a socially assistive robot for the elderly with mild cognitive impairment (MCI). This system will enable the elderly to remain active and independent for as long as possible and to also enhance their everyday quality of life. Moreover, the system should be able to adapt its behavior based on the profile of the user it interacts with (e.g., personality, preferences).

A review of the literature shows that the adaptation of the behavior of the robot can be based on the personality of the user $[15,18]$, the affective state of the user [11], or proxemics $[17,16,12]$.

There are multiple theories related to personality $[3,6,8]$. In this research, we focused on the theory proposed by Eysenck [6], as it considers that personality traits have a physiological basis. According to Eysenck's Personality theory (PEN), there are three main personality traits that characterize an individual: extraversion, neuroticism, and psychotism. Research has shown that all three personality traits are determined by biological factors [5]. In this study, we have decided to characterize the participants based only on the extraversion personality trait.

\footnotetext{
$\overline{1}$ www.enrichme.eu
} 
Dunn's Model of Sensory Processing [4] is a four-quadrant model that measures our responses to everyday sensory experiences. The model was conceptualized on the relationship between the neurological threshold (low and high), and behavioral response (accordance and counteract). In this research, we analyze only the visual and auditory sensory processing categories corresponding to all four quadrants: Sensation Avoiding (low neurological threshold and counteract), Sensory Sensitivity (low neurological threshold and accordance), Sensation Seeking (high neurological threshold and counteract), and Low Registration (high neurological threshold and accordance). The visual and auditory sensory processing categories have been chosen as they are better suited for an interaction between a robot and a user.

Individuals with high scores in Sensation Avoiding are overwhelmed or bothered by intense stimuli. Individuals with low scores do not engage in behaviors that lessen sensory stimuli. High scores in Sensory Sensitivity indicate that individuals will try to eliminate distractions. In contrast, individuals with low scores are not distracted or overwhelmed by sensory stimuli.

The purpose of this experiment is to find the interaction distance, and combination of auditory and visual stimuli in a news reading task at which the participants feel most comfortable. The personality of the participant will also be taken into account.

The present paper is structured as follows. Section 2 presents the experimental design of the experiment. The results are shown in Section 3. Finally, Section 4 concludes the paper and offers a perspective on future work.

\section{Experimental Design}

\subsection{Robotic platform and Sensors}

In this experiment, a customized TIAGo robot (see Fig. 1) was used [13], which was developed by PAL Robotics ${ }^{2}$ in Spain. The robot was customized for the needs of the ENRICHME EU H2020 project. It features a mobile base, a lifting torso, a touch-screen and a head. The frontal part of the head contains a speaker. The head features an Orbbec Astra RGB-D sensor, which provides a 640x480 RGB image at up to $30 \mathrm{~Hz}$. An Optris PI450 USB-powered thermal camera is mounted on the head of the robot. The camera has an optical resolution of $382 \times 288$ pixels and a spectral range from 7.5 to $13 \mu \mathrm{m}$. It is capable of measuring temperatures ranging from $-20^{\circ} \mathrm{C}$ to $900^{\circ} \mathrm{C}$ at a frame rate of $80 \mathrm{~Hz}$.

A Grove GSR Sensor (see Fig. 2) connected to an Arduino Uno Wifi was used to measure the skin electrical conductance of each participant.

Throughout the experiment, the movement of the robot was remotely controlled by the experimenter. 


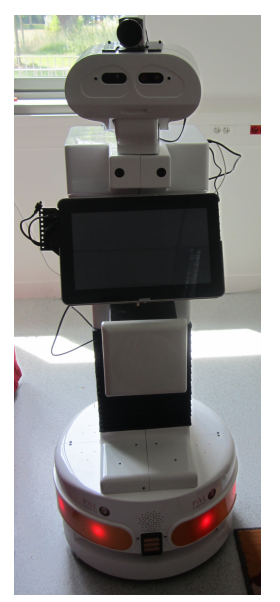

Fig. 1: TIAGo Robot

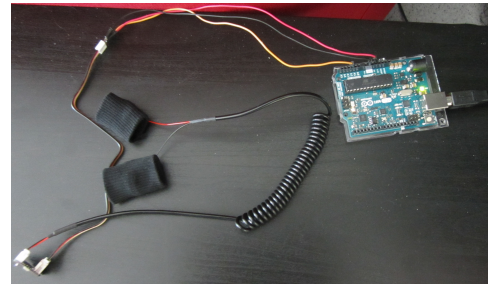

Fig. 2: Grove GSR Sensor and Arduino Uno Wifi board

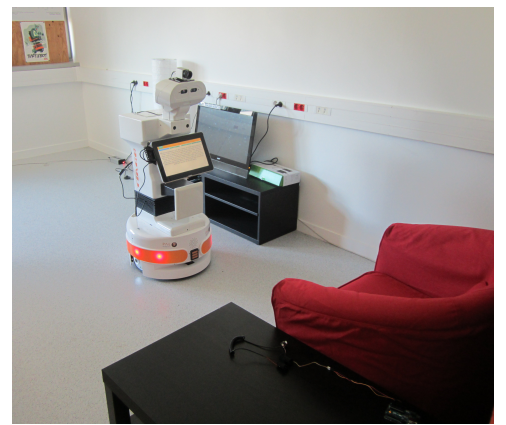

Fig. 3: Laboratory setup 


\section{$2.2 \quad$ Scenario}

Each participant was greeted by the experimenter, and then, escorted to the laboratory. In order to take part in the experiment, each participant had to sign a consent form. The Adult/Adolescent Sensory Profile (AASP) Questionnaire [2] was used to determine the sensory profile of each participant. For measuring the personality psychometric scale of Extraversion, the short version of the Eysenck Personality Questionnaire (EPQ-R) [7] was administered to the participants. Upon completing all questionnaires, the experimenter gave the participants the instructions for the experiment. Once the experimenter made sure that the participants understood what they had to do, the experiment began.

For this experiment, the ENRICHME project web-based News Application was used. The application uses the RSS 2.0 feed of different newspapers to gather the latest news and display them to the end users. The participants had to perform a news reading task. The news were displayed on the touch-screen of the robot. The robot interacted with the participants at three distances: $70 \mathrm{~cm}, 1.2 \mathrm{~m}$, and $2 \mathrm{~m}$. These distances correspond to the high limit of the personal distance, low limit of social distance, and high limit of the social distance, respectively, as defined by Hall [9]. At each of these distances the robot presents the latest breaking news to the participant. The order of the three distances is randomized.

The font size for the news was adapted based on the distance between the user and the robot. The robot detected the face of the participant, by using the Dlib toolkit [10]. Using the depth information, the robot computed the distance between its RGB-D sensor (positioned inside the head of the robot) and the face of the participant. The height of the viewport (vh) unit was used for the font size. At distances lower than $75 \mathrm{~cm}$ the font size was set to $4 \mathrm{vh}$ (see Fig. 4a ), at distances lower than $1.25 \mathrm{~m}$ it was set to $5 \mathrm{vh}$ (see Fig. $4 \mathrm{~b}$ ), and at distances greater than $1.25 \mathrm{~m}$ it was set to $6 \mathrm{vh}$ (see Fig. 4c).

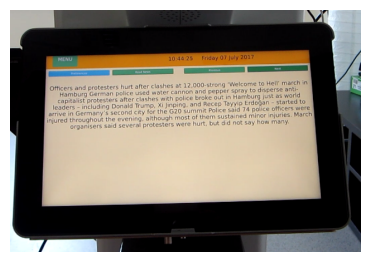

(a) Close distance $(75 \mathrm{~cm})$

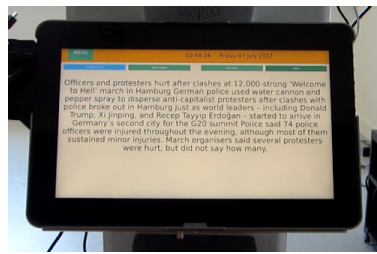

(b) Medium distance $(1.2 \mathrm{~m})$

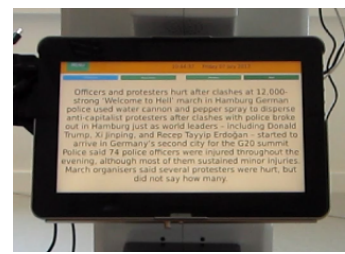

(c) Far distance (2m)

Fig. 4: Font Size Adaptation

After each condition, the participants filled-in a custom developed questionnaire. It contained multiple YES/NO questions to assess their preferences during

\footnotetext{
${ }^{2}$ www.pal-robotics.com
} 
the condition that was just finished. After each condition, we asked the participants at which distance from the robot they felt most comfortable.

\subsection{Conditions}

Based on the news reading task, we developed five conditions in which the robot used different stimuli to present the news. The robot used visual (showing or hiding the news) and auditory (read the news out loud or rest silent) stimuli. Each participant performed all five conditions in a randomized order. Each condition was made up of three phases, one corresponding to each of the three distances $(70 \mathrm{~cm}, 1.2 \mathrm{~m}$, and $2 \mathrm{~m})$.

Condition 1. Sound No Interface In this condition the robot did not display the news on the touch-screen. The news were read out loud by the robot.

Condition 2. No Sound Interface no Font Size Adaptation In this condition, the robot did not read the news out loud. The participants could read the news on the touch-screen. The font size for the news did not adapt as the robot changed the distance. A fixed font size of $6.5 \mathrm{vh}$ was used.

Condition 3. No Sound Interface Font Size Adaptation In this condition, the robot did not read the news out loud. The participants could read the news on the touch-screen. The font size for the news adapted as the robot changed the distance.

Condition 4. Sound Interface no Font Size Adaptation In this condition, the participants could both read the news and listen to them. The font size did not adapt based on the distance between the robot and the participants.

Condition 5. Sound Interface Font Size Adaptation In this condition, the participants could both read the news and hear it. The font size adapted as the robot changed the distance.

\subsection{Participants}

12 participants ( 2 female, 10 male, with mean age $\mathrm{M}=27.25 ; \mathrm{SD}=4.59$ ) agreed to take part in this experiment. Due to technical problems, the data from 2 participants had to be discarded. Therefore, the data of 10 participants was further analyzed. Table 1 shows the distribution of the participants based on their AASP results. Considering the results from EPQ-R, 4 participants are extraverted, while 6 are introverted.

\subsection{Data analysis}

During the experiment the RGB data, the thermal data, and the GSR data was recorded and analyzed offline. The RGB data was used to extract the blinks of the participants, the heart rate (HR) and the respiration rate (RR).

For the blinks, the algorithm presented in [1] was applied. First, the mean eye lid distance was computed for each of the three interaction distances. Next, for each new frame the eye lid distance is compared with the mean eye lid distance. 
Table 1: Participants distribution based on the auditory and visual sensory processing categories

\begin{tabular}{ccccc}
\hline $\begin{array}{c}\text { Sensory } \\
\text { category }\end{array}$ & $\begin{array}{c}\text { Low } \\
\text { Registration }\end{array}$ & $\begin{array}{c}\text { Sensation } \\
\text { Seeking }\end{array}$ & $\begin{array}{c}\text { Sensory } \\
\text { Sensitivity }\end{array}$ & $\begin{array}{c}\text { Sensation } \\
\text { Avoiding }\end{array}$ \\
\hline Low Visual & 6 & 6 & 4 & 3 \\
High Visual & 4 & 4 & 6 & 7 \\
Low Auditory & 2 & 8 & 2 & 4 \\
High Auditory & 8 & 2 & 8 & 6 \\
\hline
\end{tabular}

If the current distance is less than half the mean distance, the eyes are considered to be closed. If the eyes are closed for less than $500 \mathrm{~ms}$ it is considered that the participant blinked.

The method to extract the HR and RR uses the green channel of the RGB data. A Robot Operating System (ROS) [14] module was previously developed in our laboratory to extract the $\mathrm{HR}$ and $\mathrm{RR}$. The mean value of the intensity is extracted from the forehead region of the participant. The mean value was stored in a circular buffer of 10 seconds. The HR and RR could be estimated once the buffer was full. A bandpass filter was applied in order to eliminate all other frequencies which do not correspond to the HR and RR. A Hann window function was performed before a Fast Fourier Transform was applied. The HR and $\mathrm{RR}$ correspond to the index of the maximum magnitude of the frequency spectrum. The same data could be used to extract both HR and RR as the RR signal modulates the frequency and amplitude of the HR signal.

Next, a moving average filter window of 25 samples (1 second) is applied. A least-square regression is used to fit a linear model on the data. Figure 5 shows two examples of filtered data with the result of linear regression (Fig. 5a represents filtered $\mathrm{HR}$ data, while Fig. $5 \mathrm{~b}$ represents filtered RR data).

The variation of the GSR data was also analyzed. The data was filtered with a window of 30 samples (1 second). A least-square regression was applied in order to fit a model on our data. An example of filtered GSR data can be seen in Fig. 6.

\section{Experimental Results}

In our analysis, we first extracted the total number of blinks for each participant during all interactions with the robot. We performed pairwise t-tests analysis on the number of blinks by using the auditory and visual categories of all four quadrants from the sensory profile, and the personality as factors. We found no significant results when we used the personality of the participants as factor. However, when using the sensory profile, we found significant results for Low Registration auditory category type $(\mathbf{p}=\mathbf{0 . 0 1 2})$, Sensory Sensitivity visual category type $(\mathbf{p}=\mathbf{0 . 0 0 0 2 3})$, Sensation Seeking visual category type $(\mathbf{p}=\mathbf{0 . 0 0 9 2})$, 


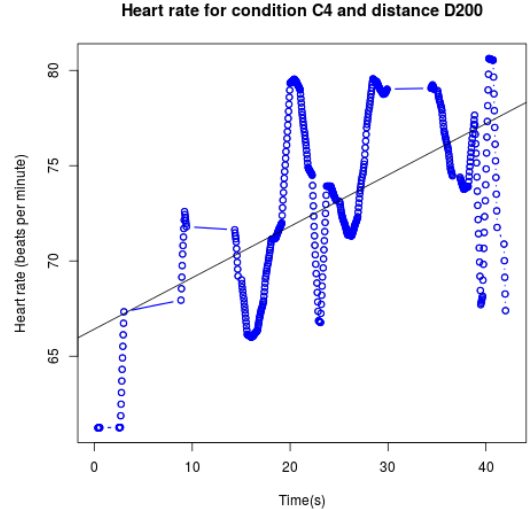

(a) Heart rate over time

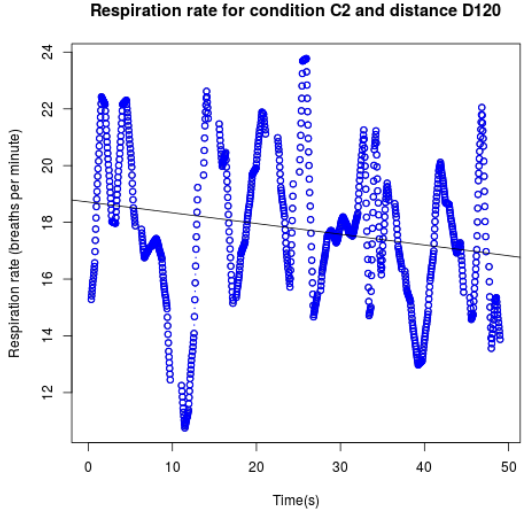

(b) Respiration rate over time

Fig. 5: Example of filtered data with the result of linear regression

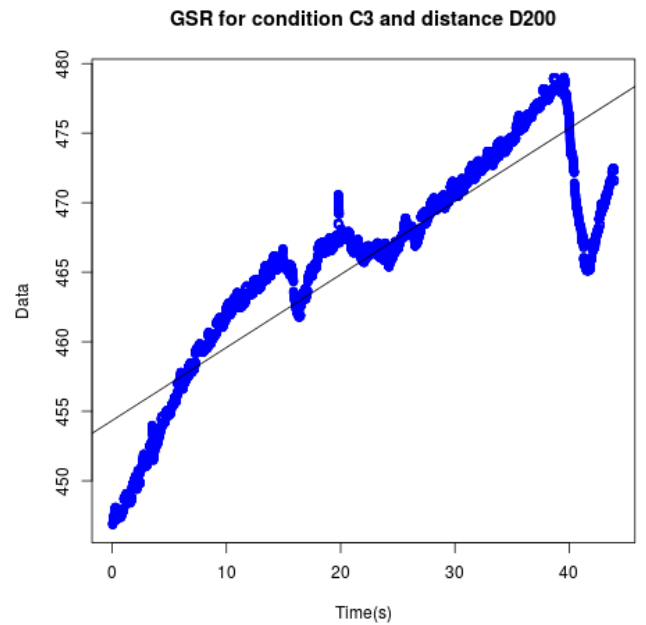

Fig. 6: GSR over time with the result of linear regression 
and both auditory and visual categories type for Sensation Avoiding (auditory $(\mathbf{p}=\mathbf{0 . 0 1})$, and visual $(\mathrm{p}=\mathbf{0 . 0 0 3 9}))$.

As we found significant results for blinking when using the sensory profile as factor, we wanted to test if the same results can be found when using the condition, the distance, and the sensory profile, or personality as factors. In order to test this, we performed multiple $5 \times 3 \times 2$ ANOVA analyses. When using the auditory processing category type significant results were found for Low Registration quadrant $(\mathrm{F}(1,105)=6.09, \mathrm{p}=0.0152)$, and Sensation Avoiding quadrant $(\mathrm{F}(1,105)=7.11, \mathrm{p}=0.008)$. For the visual processing category type significant results were found for the following quadrants: Sensory Sensitivity $(\mathrm{F}(1,105)=14.81, \mathrm{p}=0.0002)$, Sensation Seeking $(\mathrm{F}(1,105)=6.52, \mathrm{p}=0.01)$, and Sensation Avoiding $(\mathrm{F}(1,105)=8.28, \mathrm{p}=0.004)$. No significant results were found for personality.

We performed a $5 \times 3 \times 2$ ANOVA on the HR change rate as estimated by the linear model and using the condition, distance, and the sensory category for each quadrant from AASP as factors. We did not find any significant results. However, when we applied the same analysis but using personality as factor, instead of the sensory profile, we found that there is a statistically significant interaction between the condition and the personality type $(\mathrm{F}(4,105)=3.98 \mathrm{p}=0.00477)$.

We performed a $5 \times 3 \times 2$ (condition $\mathrm{x}$ distance $\mathrm{x}$ sensory category type for each quadrant) ANOVA on the respiration rate change rate as estimated by the linear model. We found significant results for Sensation Seeking visual category type $(\mathrm{F}(1,105)=4.28, \mathrm{p}=0.041)$, and for Low Registration auditory category type $(\mathrm{F}(1,105)=7.26, \mathrm{p}=0.0082)$. We did not find significant results for the analysis that uses the personality as factor.

The 5x3x2 ANOVA analysis (condition $\mathrm{x}$ distance $\mathrm{x}$ sensory category type) on the GSR change rate yielded the following significant results for the auditory category type: Sensory Sensitivity $(\mathrm{F}(1,105)=4.65, \mathrm{p}=0.0333)$, Sensation Seeking $(\mathrm{F}(1,105)=4.65, \mathrm{p}=0.0333)$. For the visual category type significant results were found only for Low Registration $(\mathrm{F}(1,105)=4.32, \mathrm{p}=0.04)$. We also found statistical significance for the personality type $(\mathrm{F}(1,105)=12.4, \mathrm{p}=0.000635)$.

The summary of the answers given by the participants for the question: "At which distance from the robot did you feel most comfortable?" is shown in Table 2 . The results suggest that most participants felt more comfortable at the close phase of social distance $(1.2 \mathrm{~m})$. This result is in accordance with the results in [17].

\section{Conclusion and Future work}

In this paper, we have presented the way in which a robot could adapt a News Application so as to provide the best combination of interaction distance, visual, and auditory stimuli, for the comfort of the individual it interacts with. We have conducted a within participant study with five conditions. The task was a news reading task, in which the participants had to read some news on the touchscreen of the robot, or to listen to some news read by the robot. The interaction 
Table 2: The distances at which the participants felt most comfortable in each condition

\begin{tabular}{cccccc}
\hline \multirow{2}{*}{ Distance } & \multicolumn{5}{c}{ Condition Condition Condition } \\
& C1 & C2 & C3 & C4 & C5 \\
\hline close $(70 \mathrm{~cm})$ & 1 & 3 & 4 & 3 & 4 \\
middle $(1.2 \mathrm{~m})$ & 6 & 7 & 7 & 6 & 6 \\
far $(2 \mathrm{~m})$ & 3 & 0 & 1 & 1 & 0 \\
\hline
\end{tabular}

between the robot and the individual took place at three distances $(70 \mathrm{~cm}, 1.2 \mathrm{~m}$, and $2 \mathrm{~m})$.

We found results that show that there is a statistically significant relationship between the conditions, interaction distance, and visual and auditory categories type for all four quadrants corresponding to AASP (Low Registration, Sensation Seeking, Sensation Avoiding, Sensory Sensitivity). We have also found significant results for the personality trait of extraversion.

Some of our future works include the analysis of the thermal data, so as to determine if the different interaction distances had an effect on the temperature variation in some regions of interest on the face.

\section{Acknowledgement}

This work was funded and done in the context of the EU H2020 ENRICHME project, Grant Agreement No: 643691

\section{References}

1. Agrigoroaie, R., Tapus, A.: Contactless physiological data analysis for users quality of life improving by using a humanoid social robot. In: Accepted in 19th Conference on Image Analysis and Processing (2017)

2. Brown, C., Dunn, W.: Adult/adolescent sensory profile: Self questionnaire. Pearson Clinical (2002)

3. Cattell, R.B., Kline, P.E.: The scientific analysis of personality and motivation. Academic Press (1977)

4. Dunn, W.: The impact of sensory processing abilities on the daily lives of young children and their families: A conceptual model. Infants and young children (1997)

5. Eysenck, H.J.: Dimensions of personality: 16, 5 or 3? - criteria for a taxonomic paradigm. Personality and Individual Differences (1991)

6. Eysenck, H.J.: Biological basis of personality. Nature (1963)

7. Eysenck, H.J., Eysenck, S.B.G.: Manual of the Eysenck Personality Questionnaire (junior and adult). Hodder and Stoughton (1975)

8. Goldberg, L.: An alternative 'description of personality': the big-five factor structure. In: Journal of Personality and Social Psychology. vol. 59 (1990)

9. Hall, E.T.: The hidden dimension (1966) 
10. King, D.: Dlib-ml: A machine learning toolkit. In: Journal of Machine Learning Research (2009)

11. Liu, C., et.al.: Online affect detection and robot behavior adaptation for intervention of children with autism. IEEE transactions on robotics (2008)

12. Mumm, J., et.al.: Human-robot proxemics: physical and psychological distancing in human-robot interaction. In: 6th International Conference on Human-Robot Interaction (2011)

13. Pages, J., Marchionni, L., Ferro, F.: Tiago: the modular robot that adapts to different research needs. International Workshop on Robot Modularity, IROS (2016)

14. Quigley, M., et.al.: Ros: an open-source robot operating system. In: ICRA Workshop on Open Source Software (2009)

15. Tapus, A., Tapus, C., Mataric, M.J.: Hands-off therapist robot behavior adaptation to user personality for post-stroke rehabilitation therapy. In: Robotics and Automation, 2007 IEEE International Conference on (2007)

16. Torta, E., et.al.: Design of robust robotic proxemic behaviour. In: Third International Conference on Social Robotics (2011)

17. Walters, M., et.al.: The influence of subjects' personality traits on personal spatial zones in a human-robot interaction experiment. In: IEEE International Workshop on Robot and Human Interactive Communication (2005)

18. Woods, S., et.al.: Are robots like people?: Relationships between participant and robot personality traits in human-robot interaction studies. Interaction Studies (2007) 